\author{
LUBLIN STUDIES IN MODERN LANGUAGES AND \\ LITERATURE 39 (1), 2015, HTTP://WWW.LSMLL.UMCS.LUBLIN.PL
}

\title{
Paul Bleton
}

5800, rue Saint-Denis, bureau 1105

Montréal (Québec) H2S 3L5, Canada

\section{La guerre telle qu'elle pourrait être}

\begin{abstract}
This paper intends to identify a representation of France at war related neither to gung-ho fiction nor to testimonial fiction in the French war novel of the first half of the $20^{\text {th }}$ century. The vein of the War-as-itcould-be will be characterized by its historical span (dating back to before WW1 and lasting long after WW2), its generic forms (future wars, espionage, melodrama...) and its ideological ambivalence (secret concerns over the French soldier's frailty and the Army's ability to adequately defend the country).

Keywords: French war novel, 1900-1939, future wars, espionage, melodrama, ideological ambivalence
\end{abstract}

À partir de la Guerre de 14, dans le roman de guerre français, la veine de la guerre-telle-qu'elle-devrait-être s'oppose à celle de la guerretelle-qu'elle-a-été, comme le bourrage de crâne à la mortifiante vérité. Par ailleurs, le discours social à propos de l'Armée faisait alors coexister deux messages, apparemment contradictoires mais fortement articulés. Le premier, celui de l' «arche sainte», de la glorification de l'Armée et de l'héroïsme militaire s'avérait homogène, surtout dans les textes scolaires. Mais le second, celui du récit construit par la culture médiatique, était plus ambigu: certes, le soldat français finissait par triompher, mais non sans que la fable ait semé le doute quant à la capacité de l'Armée de protéger le pays. 
Autrement dit, si la première opposition, la guerre-telle-qu'elledevrait-être contre la guerre-telle-qu'elle-a-été, ne manque pas de justesse, elle semble insuffisante. Telle serait la proposition à développer ici : il a existé dans la fiction de guerre une veine intermédiaire, préexistant à 1914 et se poursuivant dans l'entre-deuxguerres, sorte d'envers du Grand Récit de la France en armes, faisant la part de l'incertain et du virtuel.

\section{Guerres futures antérieures}

Le récit de guerres futures a deux grandes colorations en fait difficilement séparables, le volontarisme et l'inquiétude, la guerretelle-devrait-être et la guerre-telle-pourrait-être. L'exaltation prospective était fréquemment adressée à la jeunesse. Lorsque Georges de Lys galvanisait les énergies patriotiques des enfants des écoles par un livre de prix, L'Île envahie, racontant une tentative d'invasion de la Corse par la flotte italienne vaillamment repoussée, sauf à supposer une valeur dissuasive de cette guerre future de l'autre côté des Alpes, on est plutôt frappé par sa date de publication, 1914.

Même si les guerres futures recouraient plutôt aux ressources de la narrativité populaire, comme La Patrie en danger (1904-1905) de Paul d'Ivoi et du colonel Royet, gros in $-8^{\circ}$ de 1340 pages, il arrivait que leur récit se présente comme un document. Grandiose et austère, La Grande guerre. Russes contre Autrichiens et Allemands (1892) du lieutenant-adjudant major Émile-Nicolas Massard se recommandait par l'adéquation de la forme au propos et par la consistance des choix stylistiques. La guerre est déclarée par l'Allemagne et l'Autriche à la Russie, guerre que celle-ci gagnera à la fin. L'originalité consistait moins en cette donnée de base que dans la description journalistique des combats, la vulgarisation des rapports militaires pour que le grand public les comprenne, les illustrations de facture encyclopédique montrant, comme allaient le faire journaux et magazines pendant la Guerre de 14-18, des épisodes militaires, des pièces d'équipement, les uniformes des troupes en présence, voire les décorations accordées par les nations belligérantes à leurs héros... À cette guerre qui n'était pas encapsulée par le seul récit mais qui, éditée en grand format sur 
deux colonnes avait aussi l'apparence d'un long article d'encyclopédie, il n'aura donc manqué qu'une seule chose : d'avoir eu lieu. Paradoxe hyper-réaliste lui aussi parfaitement insolite, insuffisant pour remettre en cause l'apparente gravité de la stratégiefiction.

Auteur-vedette de Flammarion pendant deux décennies, le capitaine Danrit visait à opiniâtrement inculquer la religion de la Patrie. Un danrit était offert comme livre de prix, les parents des lycéens y voyant un complément de formation, une titillation bienvenue de la fibre patriotique de leurs rejetons. Il fut l'homme de la stratégie-fiction, genre dont le principe s'apparente au contre-factuel linguistique. Elle vise à anticiper une histoire possible ou, en modifiant un événement historique révolu, à conjecturer une histoire alternative. Cependant, ni Danrit ni les soldats qui avaient pratiqué la conjecture militaire jusqu'en 1914 n'étaient revenus sur la campagne désastreuse de 1870-71; ce genre avait surtout pour tâche de prospectivement narrativiser la Revanche. Pour Danrit, la Patrie était encore plus inépuisable que le malheur; elle pouvait indéfiniment être jetée dans de nouveaux périls, être promise à mourir, à mourir même sans pour autant susciter deuil ou culpabilité. Car toujours la mort était finalement déniée, non dans quelque esprit halluciné mais dans la réalité. Thématisant la cruauté et la mort, cette œuvre séduisait par son inépuisable vitalité; la mort y était tout aussi nécessaire dans le programme abstrait de la montée aux extrêmes qu'elle y était irréalisable - oxymore de la mort provisoire.

L'inquiétude y est à la fois palpable et bien cachée. Certes, la littérature populaire (comme chez Paul d'Ivoi), avait suivi cette voie mais avec une inspiration plus fantaisiste, avec une légèreté relative lui venant de personnages légers ou d'un optimisme technologique dynamisant. Certes, la rêverie revancharde n'était pas la seule à informer les guerres futures. Mais il n'était guère facile d'échapper à l'obsession. Dans une fiction de Rodolphe Bringer et Léon Valbert se présentant comme la suite et la conclusion de l'histoire de Fritz-laHaine (1912) se déroulant en 1870, c'est une jeune actrice qui devient ce bien improbable instrument baptisé Mamzelle-la-Revanche (1912) : 
la guerre alors encore à venir allait permettre d'effacer l'humiliante défaite de naguère.

L'éréthisme dominant et l'entrecroisement des inquiétudes nationales (dont les romans traduits alors permettent de prendre conscience) n'ont toutefois pas empêché les récits de guerres futures d'être encore exagérés, poussés à leur comble... Avec La Guerre infernale (1908) de Pierre Giffard, le genre avait même fait apparaître un pôle opposé à celui incarné par Danrit, à une polarisation de l'invention dans la virtualité martiale. Ce roman participait bien de la stratégie-fiction mais en déplaçait les accents. Le héros en était un journaliste et non un militaire; les beaux actes d'héroïsme $\mathrm{y}$ abondaient, et pourtant il s'agissait moins d'une geste de l'Armée que d'une rêverie technologique, d'un rutilant défilé du 14-Juillet de ces armes sophistiquées dont le soldat était devenu une expansion. D'un côté, les vertus militaires patriotiques étaient quintessenciées dans un patriotisme armé des Japonais, qui, même à l'époque où ils étaient associés à la France et l'Angleterre, ne laissaient pas d'inquiéter : seul un zeste d'humanisme européen empêcherait-il tout soldat de devenir un fanatique samouraï ? D'un autre côté, dépassé déjà par le comble militaire du samouraï, le soldat patriote l'était aussi par un autre comble, celui de la technologie. Celle-ci s'avérait sans patrie; par elle s'imposait la figure hyperbolique du surguerrier; les valeurs nationalistes n'ont pas prise sur lui alors que le nationalisme armé a besoin de sa compétence païenne. Le surguerrier serait ce point où la quintessence patriotique s'altère, désenchante en laissant entrevoir le complexe militaro-industriel.

Déterritorialisée par la rêverie technologique, devenue professionnelle application au combat, dédiée aux moyens plus qu'à la fin, la haine patriotique allait se recoder dans une mythologie raciste, second grand thème du roman - homicide rêverie mise au diapason des ruminations érudites d'un respectable savoir d'alors, le racisme scientifique. Elle était en outre surdéterminée par un récit modélisant, l'invasion d'Attila, et tissée d'expressions coagulées en langue, tout à fait propres à l'amplificatio rhétorique.... Une fois épuisé l'optimisme scientiste, l'avenir, déjà, n'était plus rose. 
Conviendrait-il donc de comprendre toute cette histoire comme une parodie, un renversement ironique des poncifs à la Danrit ? L'intrigue regorge d'incongruités; la panoplie qu'il y déploie ne manque ni de diversité ni d'imagination; des expressions figées réactivées soustendent l'invention... Ainsi la fiction pullule de niches pour puissants aérocars percés à flanc de montagne, d'une combinaison tactique du ballon captif et du sous-marin, d'un procédé pour dégager la nappe de brouillard au-dessus de Londres afin de ne pas être surpris par l'aéroflotte adverse, d'une arme parvenant à geler un bras du Mississipi, d'empoisonnement de l'Oural par des bacilles de choléra... Ou encore : puisque le tablier du pont gît au fond de la rivière, cent vingt soldats turcs descendent en rang dans la Bielaïa, le soulèvent et le soutiennent pendant que passe le train - modernes et martiales cariatides issues de «fort comme un Turc». Et, puisque crescendo dans l'horreur il doit y avoir, l'acmé sera atteinte par des «supplices chinois » autrement publics que ceux du jardin d'Octave Mirbeau : le médecin subit l'horrible supplice du «rat qui se promène »; une infirmière est découpée par le supplice de la «chemise de fer»; une autre ébouillantée dans celui du «serpent d'eau»; Miss Ada finit brûlée vive; Pigeon est débité en lanières puis décapité; le journaliste subit les supplices des allumettes, de la pendaison inachevée, assiste à une énucléation pour goûter par avance ce qu'il aura à subir, est finalement enterré jusqu'aux épaules, énucléé, décapité...

Lecture au premier degré ou lecture ironique ? Le mode d'emploi reste indécidable. Alors que leur matériel thématique est similaire, c'est justement par cet indécidable que La guerre infernale s'oppose le plus au monologisme de Danrit.

\section{Aimantes par l'inquiétude}

Tel serait donc le terreau de cette veine, ni guerre-telle-qu'elledevrait-être ni guerre-telle-qu'elle-a-été, issue d'une tradition romanesque antérieure à la Guerre de 14 et placée dans une insolite position en regard de la réalité historique du conflit: la guerre-tellequ'elle-pourrait-être. 
Elle allait réémerger à la fin des années 20 , alors que l'inquiétude faisait retour en de nombreux genres. À côté du thème de l'indistinction entre réalité et fiction, dans le tressage effectué par $L e$ Tsar Napoléon (1928) d'Albert Dieudonné de l'histoire de la révolution soviétique et de l'invention cinématographique - l'acteur jouant Bonaparte à Arcole serait-il Alexis-Nicolaevitch Romanov ? -, à côté de spéculations uchroniques ou anticipatrices ${ }^{1}$, les formules de la littérature populaire sont mises à contribution. Jean de la Hire reprend le thème de la fragilité coloniale dans Le Secret des cent îles (1929), mais aussi celui de la fragilité de la concorde sociale dans L'Homme aux hélicoptères (1928). Sa science-fiction imagine, dans L'Île d'épouvante (1930), l'affrontement titanesque, manichéen et économique entre deux blocs du XXII ${ }^{\mathrm{e}}$ siècle, chacun dirigé par une " société de journalisme, de propagande et de publicité », l'Universel de Saint-Clair, dit «le Nyctalope », héros sériel de de la Hire, et le Mondial de l'ignoble Mézarek, fils de Zattan et de Titania la princesse rouge. Sans la noblesse du capitaine Nemo, Feodor Sarraskine, le sombre héros de La Guerre des océans (1928) de José Moselli, poursuit la tradition du vengeur sous-marin de Verne. Il partage un même imaginaire technologique populaire avec La Croisière du tank sous-marin (1931) de Guy Péron et brode sur le thème d'un pouvoir malveillant et caché qu'illustrent les six volumes des Samouraïs $d u$ Soleil pourpre (1931) d'Albert Bonneau - roman d'aventures donnant une variation "société secrète » à l'idéologème du péril jaune. $L a$ Ruée des jaunes (1933) de Louis Gastine ramène à l'anticipation martiale, sans pour autant se détacher du topos du vengeur, ici le Maître de la cité secrète d'Hitor construite en plein désert de Gobi, descendant de Tamerlan, qui, profitant de l'affaiblissement politique et moral d'un Occident débilité par la "peste démocratique », fait avancer 300 millions d'Asiatiques contre l'Europe - comble de la

\footnotetext{
${ }^{1}$ Comme La Der des der, roman de la prochaine guerre (1928) de Victor Méric, Si Napoléon en 1914... (1930) de Jean-Marie Bourget, Ciel rose (1933) de Michel Corday, Toscins dans la nuit (1934) de Willy-A. Prestre, La catastrophe (1936) de Henri Baraude, Victoire à Waterloo (1937) de Robert Aron.
} 
guerre de masse - après avoir assujetti les Soviets sans qu'ils en aient eu conscience.

À la fin des années 30, la spéculation sur la guerre future ne nécessitait plus grand effort d'invention et l'indécidable ironie à la Giffard n'était plus de saison. La possible ambivalence des guerres futures était rapidement neutralisée et, sous les oripeaux du virtuel le lecteur reconnaissait rapidement la guerre-telle-qu'elle-devrait-être. Dans le droit-fil de son argumentaire anti-parlementaire, Ciel de feu (1934) de Léon Daudet imagine une invasion de blindés ayant pour objectif immédiat moins l'occupation du territoire que l'accès à l'Atlantique pour installer des bases de sous-marins. Que l'on se rassure : l'énergie des héros saura réagir, en ne s'en laissant surtout pas conter par les sophismes des politiciens. Sans doute que l'effort allemand est puissant sur les lignes de défense françaises, mais pas assez pour inquiéter la solide ligne Maginot ou prévenir l'action de la cavalerie motorisée et de sa légèreté aventurière - thèmes des deux volumes de Face à l'ennemi (1939) du commandant Verdun, dont la fonction de bouchon à angoisse est confirmée par la signature préfacielle du général Weygand.

Sans parvenir à la sombre flamboyance de ceux de l'avant-guerre, les romans populaires thématisant de probables guerres contre l'Allemagne laissent beaucoup mieux filtrer cette angoisse. Elle prend deux véhicules thématiques déjà bien campés dans l'imaginaire du genre: la rêverie inquiète sur les technologies de guerre et la dénonciation alarmée de l'efficacité de l'espionnage.

Albert de Pouvourville allait disposer de deux séries-gigognes chez Baudinière, «La Guerre prochaine » et «L'Héroïque aventure », plus portées vers le premier de ces imaginaires. Encore plus technofictionnelle, dans ses romans $\grave{A}$ deux doigts de la fin du monde (1928) et Le tunnel de Gibraltar (1933) ou dans sa série fasciculaire 193? La guerre est déclarée (1931-1932), l'inspiration du colonel Royet laisse déjà une part à l'espionnage et à un imaginaire du secret et de la dissimulation. Plus fidèle à l'inspiration de Jean de la Hire ${ }^{2}$, la série

${ }^{2}$ C'est lui qui se dissimulait derrière le nom de plume de commandant Cazal. 
La guerre! la guerre! (1939) du commandant Cazal, pourtant tout à fait militaire, n'en traitait pas moins d'espionnage. Chaque fois, le roman prétend à une paradoxale vraisemblance (celle d'auteurs informés des dessous de la guerre secrète) et adopte une conception de l'espionnage moins manichéenne que dans la période précédente.

\section{La fragilité du soldat}

Guerre de masse, guerre de ruse, guerre technologique : ce qui risque d'advenir à la France, de l'extérieur, et de l'emporter, se double d'un risque plus intime au sein même de la force nationale. Sans que son courage ou son abnégation soient d'habitude mis en cause, le soldat censé protégé le pays est-il à la hauteur de sa tâche ?

Entendons bien que ce ne sont pas les attaques de la tradition antimilitariste (Abel Hermant, Lucien Descaves, Georges Darien, etc.) qui sont visées ici. Dans la tradition du mélo, le soldat-victime de Jules Mary était confronté à une malveillance radicale mais, jusqu'à la Guerre de 14, sans réelle articulation institutionnelle. Dans le roman d'espionnage, c'est structuralement que cette fonction du soldat victime s'intégrait dans les lois du genre en émergence, exploration d'une plus inquiétante facette de la fragilité du soldat français. Ce genre, dont la visibilité s'était accrue dans les années précédant 1914 par le nombre de titres et la célébrité des romancier ${ }^{3}$, démontrait l'ignominie et l'efficacité d'une arme considérée avec horreur par le bushido saint-cyrien, ce qui donnait un avantage à l'officier prussien, honoré de servir comme espion. Chez Mary, à l'inhumanité (des armes chimiques et biologiques anticipées dans Le Boche empoisonneur) devait répliquer la ruse; l'articulation avec le thème du soldat victime se faisait chez lui aussi sur une base de classe sociale : c'est d'habitude le riche, le bourgeois, l'officier qui pâtissait et le paysan, l'homme du peuple qui maniait feintes, stratagèmes et contrefourberies.

\footnotetext{
${ }^{3}$ Des gros canons de la littérature populaire, Arthur Bernède, Aristide Bruant, Paul d'Ivoi, Georges Le Faure, Gustave Le Rouge, Jules Mary, Pierre Souvestre et Marcel Allain...
} 
Les intrigues de martyr féminin «nationalisaient » leurs victimes traditionnelles avec la figure de l'Alsacienne. La pauvre Alsacienne déplacée, accusée d'espionnage à cause de son accent, dans L'Espionne du Bourget de Paul Bertnay (1909), incarnerait le type de la victime civile de l'annexion. Or, plus adapté à une longue durée, ce type allait s'alimenter à l'une des traditions majeures de la littérature populaire, le roman de la victime. Ainsi, longtemps après la défaite, Marie-Thérèse, la jeune Alsacienne de Bruant, aime Frantz d'un amour impossible : son père, un industriel vénal, prêt à sacrifier sa fille pour sauver son usine, veut la forcer à épouser un officier de l'armée d'occupation. Frantz, lui-même Alsacien, en service militaire chez les uhlans, va rendre visite à Marie-Thérèse dans le jardin familial où le lieutenant Karl Wangan les surprend enlacés. Les deux hommes en viennent aux armes, Frantz est blessé, proscrit : c'est le début de deux volumes de tribulations.

Si Maurice Leblanc avait déjà sacrifié à la veine revancharde avec La frontière (1911), avec L'éclat d'obus (1915), la fantasque légèreté anarchiste de Lupin prend une gravité nouvelle, son ingéniosité investigatrice et manipulatrice est mise au service de la France. Et dans Le Triangle d'or (1917), L'Île aux trente cercueils (1919) et Les Dents du tigre (1920), sans être appelé ni fonctionnarisé, le voici contre-espion, amateur mais inspiré. Comment ne pas voir dans ce recours au héros exceptionnel une autre forme de l'ambivalence affectant les guerres futures, symptôme de la fragilité du pays ?

Indice du retour de l'inquiétude, les années 30 voient non seulement l'accroissement de la quantité de titres, mais aussi l'émergence de quelques œuvres marquantes de la fiction d'espionnage $^{4}$ et de séries ${ }^{5}$, la multiplication de romans d'espionnage

\footnotetext{
${ }^{4}$ Le succès de Jean Toussaint-Samat, de Jean Bommart, de Pierre Nord, de Pierre Apestéguy.

5 «La guerre secrète du contre-espionnage. Les aventures de Jean-Marie Le Couldrier » de Jean Toussaint-Samat, «Ceux du S.R.» de Charles Robert-Dumas, «Thérèse Arnaud, espionne française », série fasciculaire de Pierre Yrondy.
} 
dans la collection du Masque, les premières collections spécialisées ${ }^{6}$ et, en général, l'instauration d'un continuum plutôt que le respect d'une frontière entre information investigatrice et invention romanesque. La Drôle de guerre, la défaite et l'Occupation allaient beaucoup réduire cette production romanesque, sans la tarir tout à fait; mais, sauf pour Pierre Nord, dès la fin des années 40, l'espionnage sérialisé s'était éloigné de la thématique de la fragilité du militaire.

4. En somme...?

L'originalité de cette modalité de la-guerre-telle-qu'elle-pourraitêtre aura consisté à articuler les thèmes de la gloire de l'Armée et de l'inquiétude, du virtuel et de l'incertain, de la valorisation d'une Armée si organiquement liée à la société que le futur du pays peut reposer entre ses mains et d'un scepticisme quant à son efficacité protectrice, scepticisme bien plus délétère parce que moins ostensible, moins frontal que dans les traditions antimilitaristes et pacifistes. L'inquiétude exprimée là se fondait dans le doute que le conservatisme de l'Armée ait su évaluer avec justesse les mutations de l'art de la guerre et ait su entreprendre la nécessaire modernisation de la conception de l'honneur militaire médiéval face à l'arme de l'espionnage et à la technologisation du combat.

\section{Bibliographie}

Aron R. (1968 [1937]) : Victoire à Waterloo. Paris : Plon.

Baraude H. (1939 [1936]) : La Catastrophe. Paris : Eugène Figuière.

Bertnay, P. [P. Breynat] (1939 [1909]) : L'Espionne du Bourget. Paris : A. Fayard.

Bonneau, A. (1931): Les Samouraïs du Soleil pourpre, 1. Les Samouraïs du soleil pourpre, 2. Les mystères de Chinatown, 3 Les damnés de Sakhaline, 4. La jonque au cercueil, 5. Le trésor du shogun, 6. La reine du hara-kiri. Paris : Tallandier.

Bourget J.-M. (1930) : Si Napoléon en 1914... Paris : Gallimard.

Bringer R., \& Valbert L. (1912) : Fritz-la-Haine, roman d'aventure et Mamzelle-laRevanche. Paris : Méricourt.

${ }^{6}$ Mémoires de guerre secrète de la Librairie des Champs-Élysées et Guerre secrète de Baudinière. 
Cazal cmdt [J. de la Hire] (1939): 1. La guerre! la guerre!, roman de demain, 2. Maginot-Siegfried, 3. Batailles pour la mer, 4. L'Afrique en flamme, 5. La fin par le pétrole. Paris : Tallandier.

Corday M. (1933) : Ciel rose. Paris : Flammarion.

Danrit cpt [É-C. Driant] (1895) : La Guerre au vingtième siècle. L'Invasion noire - 1. La mobilisation africaine, 2. À travers l'Europe. Paris : Flammarion.

Danrit cpt. (s.d [1895]) : La Guerre de demain. 1. La Guerre des forts, 2. La guerre en ballon, 3. La guerre en rase campagne,4. Le journal de guerre du lieutenant von Piefke. Paris : Fayard.

Daudet L. (1934) : Ciel de feu, Paris : Flammarion.

Dieudonné A. (1928) : Le Tsar Napoléon, Paris : Baudinière.

Gastine L. (1933) : La Ruée des jaunes. Paris : Baudinière.

Giffard P. (1908) : La Guerre infernale, janvier-août (30 fascicules ; ill. A. Robida). Paris : Méricant.

La Hire J. de. (1928) : L'Homme aux hélicoptères, Paris : Tallandier.

La Hire J. de. (1929) : Le Secret des cent îles, Paris : Tallandier.

La Hire J. de. (1930) : L'île de l'épouvante, Paris : Fayard.

Royet col., \& d'Ivoi P. (1904-1905) : La Patrie en danger. Paris : Geffroy.

Leblanc M. (1911) : La Frontière. Paris : Lafitte.

Leblanc M. (1916) : L'Éclat d'obus. Paris : Lafitte.

Leblanc M. (1917) : Le Triangle d'or. Paris : Lafitte.

Leblanc M. (1919) : L'Île aux trente cercueils. Paris : Lafitte.

Leblanc M. (1920) : Les Dents du tigre. Paris : Lafitte.

Lys, G. de [G. de Bonnerive] (1914) : L'Île envahie. Tours : Mame.

Mary J. (1914) : Le Boche empoisonneur. Paris : Tallandier.

Massard lt-adj. major É-N. (1892) : La Grande guerre. Russes contre Autrichiens et Allemands. Paris : Fayard.

Méric V. (1928) : La Der des der, roman de la prochaine guerre. Paris : Éditions de France.

Moselli J. (déc. 1928-sept. 1929) : «La guerre des océans ». Sciences et voyages, $\mathrm{n}^{\circ}$ 485-524.

Péron G. (1931) : La Croisière du tank sous-marin. Paris : Tallandier.

Pouvourville A. de. (1934-1935): La Guerre prochaine (5 fascicules). Paris : Baudinière.

Pouvourville A. de. (1939) : L'Hérö̈que aventure, (25 fascicules). Paris : Baudinière.

Prestre W-A. (1935) : Toscins dans la nuit. Neuchâtel, Paris : Attinger.

Robert-Dumas C. (1934) : Deuxième bureau. Paris : Fayard. 
Robert-Dumas C. (1934) : Les Loups entre eux. Paris : Fayard.

Robert-Dumas C. (1934) : L'Homme à abattre. Paris : Fayard.

Robert-Dumas C. (1939) : L'Embardée. Paris : Fayard, 1935.

Robert-Dumas C. (1939) : L'Idole de plomb. Paris : Fayard, 1935.

Robert-Dumas C. (1936) : L'Usine fatale. Paris, Paris : Fayard.

Robert-Dumas C. (1938) : La Marque du triangle, Paris : Fayard.

Robert-Dumas C. (1939) : Agent double. Paris : Fayard.

Robert-Dumas C. (1947) : L'Embauche. Dillberger contre Benoit. Paris : Fayard.

Royet col. [M. Colroy] (1928) : À deux doigts de la fin du monde. Paris : Ferenczi.

Royet col. (1931-1932) : La guerre est déclarée, (20 fascicules). Paris : Tallandier.

Royet col. (1933) : Le Tunnel de Gibraltar. Paris : Tallandier.

Samat J-T. (1934) : Des Espionnes nues. Paris : Baudinière.

Samat J-T. (1934) : L'Espionne au corps de bronze. Paris : Baudinière.

Samat J-T. (1935) : La Douce-Vierge-de-la-Merci. Paris : Baudinière.

Samat J-T. (1935) : Trinidad, simple barque. Paris : Baudinière.

Samat J-T. (1936) : Déserteur et sous-marins. Paris : Baudinière.

Verdun cmdt. (1939): Face à l'ennemi. 1. La guerre souterraine, 2. L'escadron Cyclone. Paris : Denoël.

Yrondy P. (1934-1936) : Thérèse Arnaud, espionne française (65 fascicules). Paris : Baudinière. 